\title{
Class-II division-I malocclusion treated with extraction of maxillary first premolars- a case report
}

\author{
Jahan $\mathbf{H}^{1}$ BDS, FCPS, Hossain MZ ${ }^{2}$ BDS, PhD and Rahman $\mathbf{M M}^{3}$ BDS, FCPS
}

\begin{abstract}
This case report describes the treatment of a 13 years old girl named Fahrial Sharmin came to Dhaka Dental College with complaints of moderate maxillary proclination. She had class-II molar and canine relationship and showed $12 \mathrm{~mm}$ overjet and $5.5 \mathrm{~mm}$ overbite with lip trap. Treatment started with extraction of maxillary first premolars and fixed orthodontic therapy. Treatment were finished in a class-II molar relationship with canine guidance and ideal overjet $\&$ overbite relationships were established. The final result was esthetically well balanced.
\end{abstract}

Keywords: Class-II division -1 malocclusion, Protrusion, Maxillary premolar extraction.

\section{INTRODUCTION}

A class II intermaxillary dental relationship represents a posterior discrepancy of the lower teeth with regard to the upper teeth. The embrasure between the lower canine and the lower first premolar is shifted backward with regard to the upper canine (blue arrows). The center of the lower first molar (mesiobuccal groove) is posterior to the first corner (mesiobuccal cusp) of the upper first molar (red arrows) The overjet reflects the anteroposterior discrepancy (yellow arrow ) and the overbite is increased and the lower teeth are hidden completely by the upper teeth and touch the gingiva at the back of the upper teeth (green arrow).
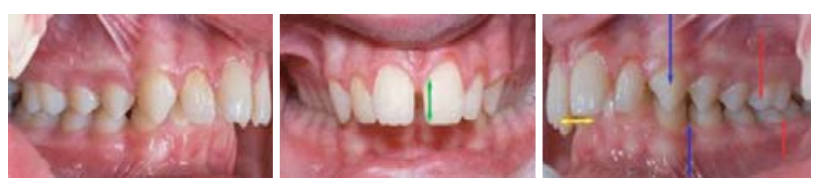

Class-II division-1 malocclusion is the most common malocclusion encountered in Bangladesh. The treatment of skeletal class-II division-1 malocclusion depends upon the age of the patient, growth potential, severity of malocclusion, and compliance of patient with treatment. There are three possible approaches to treat a skeletal class-II malocclusion.

1.Modification of growth with myofunctional appliance . 2.Camouflage treatment with extraction either 2 or 4 premolars (displacing the teeth to obtain proper functional occlusion despite the skeletal discrepancy).

3.Surgical repositioning of the jaw. ${ }^{1}$

But in adult cases, where growth no occours, camouflage \& surgery are the only treatment option. Which of these is the best approach is highly controversial.The effects of surgical treatment versus camouflage treatment can be measured in terms of the clinician outcome, i.e. the changes in dental occlusion, cephalometric measurement, and esthetic changes that occur. Where comparing the alternative treatment plans, it also is important to evaluate treatment efficiency, determined by whether and to what extent the treatment goals were met by improving dental relationships and dentofacial esthetics. $^{2}$ One of the camouflage options available is the extraction of the maxillary premolars, correcting the canines to a normal class-I relationship, leaving the molars in a class-II relationship. Treatment with 2 premolars extraction gives a better occlusal result than treatment with 4 premolars extraction. ${ }^{3}$

This case reports a 13 years female patient having Angles Class-II division-1 malocclusion treated with extraction of maxillary first premolars.

Pre-treatment Assessment:

Name-Fahrial Sharmin

Age -13 yrs

Patient complained of forwardly placed teeth \& inability to close the lips \& no relevant medical history. Extra-oral examination revealed convex profile $\&$ incompetent lip with lip trap.

Intra-oral examination revealed class-II molar \& canine relationship, good oral hygiene, healthy soft tissue, increased overjet \& overbite.

OPG examination revealed all permanent teeth were present except second \& third molars. (unerupted)

Cephalometric examination revealed class-II skeletal pattern with moderate maxillary incisor proclination \& average growth pattern.

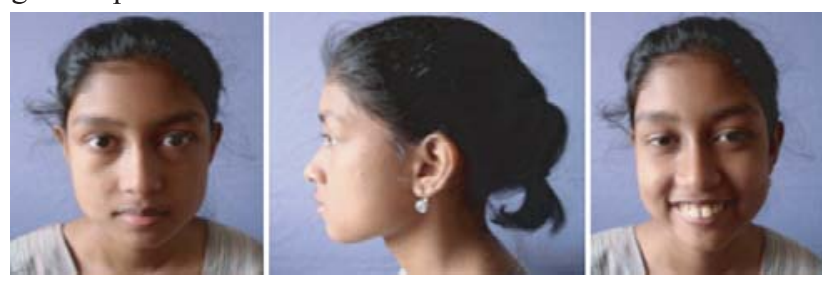

Pre - treatment extraoral frontal \& profile photographs

${ }^{1}$ Dr. Hasnat Jahan BDS, FCPS, Lecturer, Dept of Orthodontics ,Dhaka Dental College and Hospital. ${ }^{2}$ Prof. Dr. Md. Zakir Hossain BDS, PhD, 

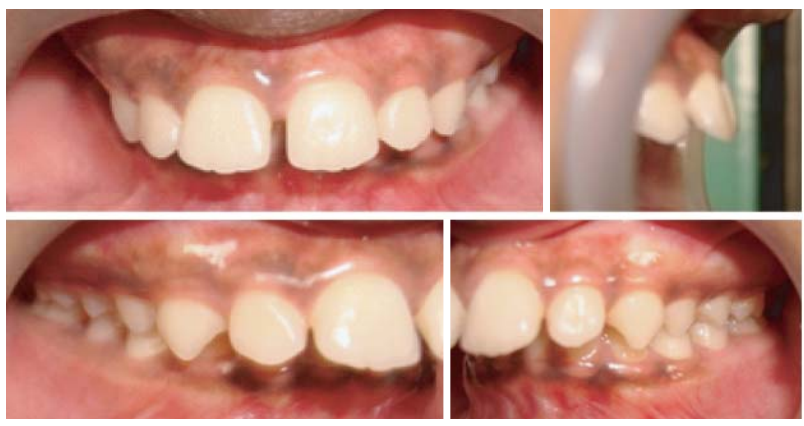

Pre reatment intra-oral photographs

Treatment Objectives:

Retraction of maxillary anteriors

Establishment of normal overjet \& overbite.

Improved facial profile.

Achieve class-I canine relation if extraction of only maxillary premolars.

\section{Treatment Plan:}

Extraction of maxillary first premolars.

Alignment \& levelling of both arches.

Canine retraction of maxillary arch.

Arch contraction of upper arch.

Arch co-ordination \& interdigitation.

Retention.

Post- treatment Assessment:

Class-I canine relation

Straight profile

Normal overjet \& overbite

Duration of the treatment was 2 years.

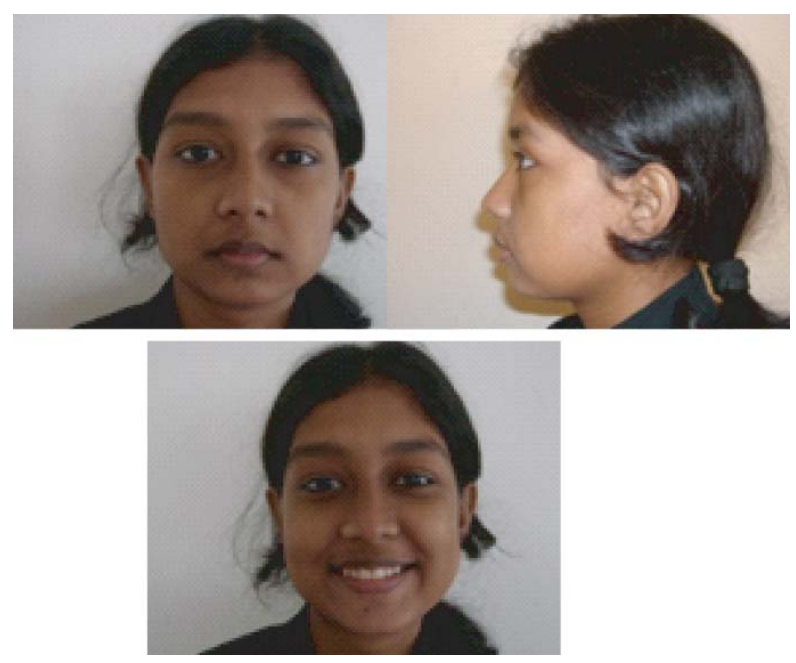

Post treatment extra-oral smiling

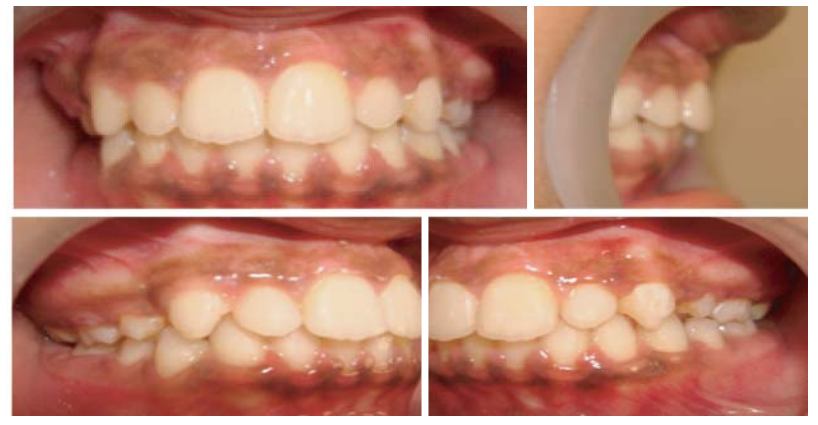

Post treatment intra-oral photographs

\section{DISCUSSION:}

Patient was improved with smile \& profile. Upper incisors were retracted to achieve normal incisor angulations, overjet $\&$ overbite. Bilateral class-I canine relation was achieved, molar relation was class-II (extraction of only upper first premolars), lower lip exhibited normal position in relation to E-line. The case was successfully managed by extraction of maxillary first premolars \& fixed appliance using Standard Edgewise Technics.

\section{CONCLUSION:}

Successful treatment required a careful diagnosis, specific planning, and efficient biochemical control of the force systems to correct the disharmony in the vertical \& horizontal dimensions.

\section{REFERANCES:}

1. Proffit WR, Philips C, Dann C. Who seeks surgical orthodontic treatment? Int J Adul Orthod Orthogn Surg. 5:153-60, 1990.

2. Proffit WR, Philips C;Douvartzidis N. A comparison of outcome of orthodontics \& surgical orthodontic treatment of class-II malocclusion in adult . Am. J. Orthod. 101: 556- 565, 1992.

3. G Janson, AC Brambilla, JFC Henriques, MR de. Class-II treatment success rate in 2- and 4- premolar extraction protocols, Am.J.Orthod. 125 (4) :472-479, 2004.

\section{Correspondence}

Dr. Hasnat Jahan BDS, FCPS, Lecturer, Dept of Orthodontics, Dhaka Dental College and Hospital. Cell : +88 01758943130

E-mail : drhasnatjahan@gmail.com 